\title{
(INTRINSIC) VARIATIONS OF WOLF-RAYET STARS
}

A.M. van Genderen ${ }^{1}$, M.A.W. Verheijen ${ }^{1}$, E. van Kampen ${ }^{1}$, F.H.A. Robijn ${ }^{1}$, R. van der Heiden ${ }^{1}$, B.P.M. van Esch ${ }^{1}, H$. Greidanus $^{1}$, R.S. le Poole ${ }^{1}$, R.A. Reijns ${ }^{1}$, K.A. van der Hucht $^{2}$, H.E. Schwarz ${ }^{3}$, C.W.H. de Loore ${ }^{4}$, E. Kuulkers ${ }^{5}$, L. Spijkstra

${ }^{1}$ Leiden Observatory, Postbus 9513

2300 RA Leiden, The Netherlands

${ }^{2}$ SRON Space Research Utrecht, Sorbonnelaan 2

3584 CA Utrecht, The Netherlands

${ }^{3}$ ESO, Casilla 19001

Santiago 19, Chile

${ }^{4}$ Astrophysical Institute, Pleinlaan 2

1050 Brussels, Belgium

${ }^{5}$ Astronomical Institute, Roetersstraat 15

1018 WB Amsterdam, The Netherlands

\section{ABSTRACT}

Twenty - two Wolf-Rayet stars (12 of type WN and 10 of type WC) were observed in the years 1986-1990 with the VBLUW photometer of Walraven. Eight (WC and WN) objects appeared to be constant. Five of the in total 14 variable objects will be discussed in the present paper: WR46 (WN3 pec), WR50 (WC6+a), WR55 (WN8), WR86 (WC7) and WR123 (WN8).

Simultaneous spectroscopy is made for WR46 and WR50. These results will be also discussed in short.

\section{Introduction}

This paper, based on the oral contribution at the IAU Symposium No. 143, has a title which probably suggests more than can be justified by the authors. Consequently the reader might see his expectations for an enlighting description on "intrinsic variations" (thus directly caused by the photosphere) blighted.

K. A. van der Hucht and B. Hidayat (eds.),

Wolf-Rayet Stars and Interrelations with Other Massive Stars in Galaxies, 129-145.

(c) 1991 IAU. Printed in the Netherlands. 
Nevertheless the various morphological types of light curves are most interesting and still not quite understood.

Although duplicity among WR stars appears to be an important source of variability (Vreux, 1987; Moffat and Shara, 1986) and many WR stars turn out to be constant, we have not given up the hope that real intrinsic variations, thus directly reflecting photospheric phenomena, once might be established for some of them. A crucial condition is that the suspected specimen (if any) should be observed intensively by various techniques.

If random or large scale turbulence in the envelope causes strong emission line variations (see for example the review paper of Vreux 1987), this could turn up as small light variations if medium broad band photometry is applied. However, they are not the "first order" type of variations we are looking for. They rather could be called variations of the "second order".

We shall discuss here in short five peculiar specimens of variable WR stars. A more detailed analysis will be given in forthcoming papers.

\section{Observations and reductions}

The observations of the objects listed in Table 1 of which some will be discussed here were made with the $90-\mathrm{cm}$ Dutch telescope at the ESO, La Silla, Chile, by various observers during the interval 1986-1990. The telescope is equipped with the VBLUW simultaneous photometer of Walraven. The last detailed description of this system is given by Lub and Pel (1977).

Each object was alternately measured relative to a nearby comparison star a number of times per night, or during a few hours in a row, or during complete nights. The diaphragm aperture was 16". Typical integration times per measurement varied between 1 and 5 minutes. During the time that an object was monitored, the time resolution varied between 5 and 15 minutes. Calibrations and corrections for differential extinction were applied with the aid of standard stars measured throughout each night.

Twice the average standard deviation $(2 \sigma)$ per data point is indicated in the figures representing light and colour curves (in log intensity scale). All these curves are relative to the comparison star.

For some objects also simultaneous spectroscopy was performed by monitoring them for several hours with the $B \& C$ spectrograph of the ESO $1.52 \mathrm{~m}$ telescope equipped with a CCD detector. The resolution $R=\frac{\lambda}{\lambda} \sim 450$, spectral range: 4080-7150 A. Part of the calibration and reduction is still underway, part of it will be discussed in the present paper.

\section{The objects under investigation}

Table 1 lists the 22 WR stars observed by our group during the 
Table 1. The WN and WC type Wolf-Rayet stars, indicated by their WR numbers (van der Hucht et al., 1981), investigated by our group with multi-colour photometry. Underlined objects are variable. objects marked with an asterisk were partly simultaneously monitored with spectroscopy.

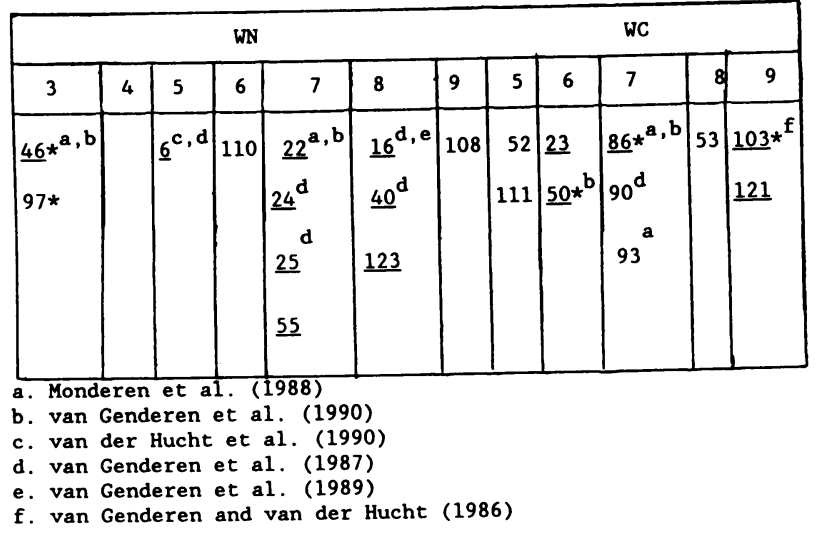

last four years and still only partly published. Most of the objects were monitored during a number of hours per night, sometimes within a time span of one month, others in a few months and scattered in two or more seasons.

The sample consists of $12 \mathrm{WN}$ type and $10 \mathrm{WC}$ type stars. Nine specimen of the first group and five of the second group are variable. It must be noticed that this is no reliable statistic, since many stars were observed because they were known variables already. Others were simply put on the program just to investigate whether they are variable.

It is of interest to mention that eight objects turned out to be constant. That is to say that the noise is usually smaller than $0^{\mathrm{m}} 005$ (differentially with respect to a nearby comparison star).

A glance at Table 1 shows that there is obviously no correlation between variability and spectral type. Obviously constancy is a normal habit among an important part of the WR stars. This does not exclude the possibility that the envelope masks any variation of the photosphere.

4. The 1ight-and colour curves of a selected sample

\subsection{WR46 $=$ HD104994 (WN3 PEC)}

WR46 $\left(\mathrm{V}_{\mathrm{J}} \sim 10.9\right)$ was observed relative to $\mathrm{HD} 108355$ (B8IV, $\mathrm{V}_{\mathrm{J}}$ 6.0) in February and March 1989 by two of us (E.K. and E.v.K, respectively). 
Typical light and colour curves for one night are shown in Fig. 1 .

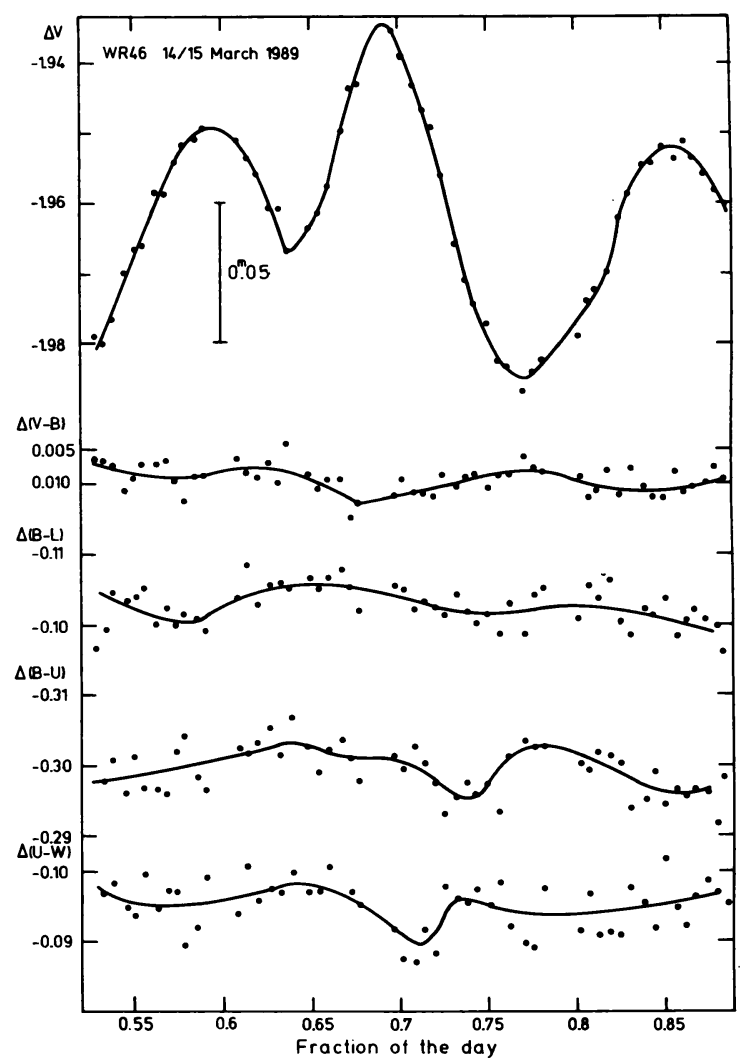

Fig. 1 Typical light and colour curves of WR46 (WN 3 pec) (Observations by E.v.K.)

The brightness and colours relative to the comparison star are plotted as a function of H.J.D.

The time scale of a single wave is $\sim 3.3 \mathrm{~h}$, the same as for the observations in 1986 (Monderen et al., 1988) and in 1988 (van Genderen et al., 1990). The light curve often shows per night one deep and one shallow minimum. The maxima do not differ always in height and sometimes bumps are present on one of the branches.

The period search program of Sterken (1977) was used in the range $0^{d} 1$ and $0^{d} 4$ and with steps of $0^{d} 0001$ and $0^{d} 001$. For both sets (February and March) the best candidate period is $0^{d} 1412 \pm$ $0^{d} 0002$, resulting in a one peaked light curve with a large scatter due to the varying shape of each cycle. However the phase diagram for a period twice this length: $P=0^{d} 2824$ is shown in Fig. 2 
(March 1989 observations only), because of the significant difference between the two maxima of the colour curves $V-B$ and $V$ $W$ (we omit the $U-W$ curve). It could mean that this period is more significant than the previous one. The scatter is of the same order.

If the February 1989 data set is combined with those of March, the scatter in Fig. 2 slightly increases, but apparently the period is still valid. Whether it is stable over an interval of years cannot be checked due to the large time gap between the observations of 1986,1988 and the present set (1989).

The light curve resembles that of a binary consisting of a deformed bright component (the WR star) and a smaller one. The average distance between the extrema is $\sim 0.25$ of the period, which is in favour of the binary hypothesis. However, the variation in the times of the extrema varies sometimes by $15 \%$ or more. If the binary hypothesis is correct, this variation must be caused by some other effect superimposed on the binary modulation. An analysis of the height variation of the extrema seems to indicate a more or less systematic trend with a periodicity of $\sim 0^{d} 30$, which is slightly longer than the binary period. Whether this periodicity is real cannot be said due to the relative short time interval in which the March data are collected (eight nights). (The February data set is a much smaller data set and therefore not used). One could think of luminous clouds in a disk around the system rotating slightly slower than the binary, which cause the extra modulation, or we are dealing with a (randomly) varying shape of the continuum emitting region. The peculiar fact that the colours are bluer in the light minima, instead of redder as it should be in the case of normal elongated stars, might be caused by a better transparency of the envelope of the WR star at the place facing the companion and at the opposite side. Due to the lower gravity at the points of a normal distorted star, the temperature should be lower there.

A period of $0^{d} 28$ or $6.8 \mathrm{~h}$ is very short for a massive binary. With Kepler's law and adopting total masses of $5,10,15,20$ and $40 \mathrm{M}_{\odot}$ we find for the distances between the components $3.1,3.9$, $4.4,4.9$ and $6.2 R_{\Theta}$, respectively. This is not impossible since the radius of WN type stars can be as small as 0.5-5 $R_{\theta}$ (Cherepashchuk et al., 1984; Hillier, 1987; Hamann et al., 1988; Langer, 1989). Then $\mathrm{V}_{\text {orbit }}$ amounts to $520,650,700,800$ and 1000 $\mathrm{kms}^{-1}$, respectively.

The total mass of the system is estimated as follows:

$\mathrm{M}_{\mathrm{v}}(\mathrm{WN} 3)=-2.8, \mathrm{BC}=-4.2 \pm 1.2$ (van der Hucht et al., 1988), we find $M_{b o l}=-7.0 \pm 1.2$. Then with the aid of the M-L relation of Maeder and Meynet (1987): $M=3.2(+4.1,-1.8) \mathrm{M}_{\Theta}$. Consequently, the total mass of the system should lie somewhere in the range $1.4-7.3 \mathrm{M}_{\Theta}$.

The simultaneously obtained spectroscopic observations in three nights in March 1989 by one of us (H.E.S.) are partly analyzed and allow some quantitative discussion. Each night about 
40 spectra were obtained with integration times of $3 \mathrm{~min}$.

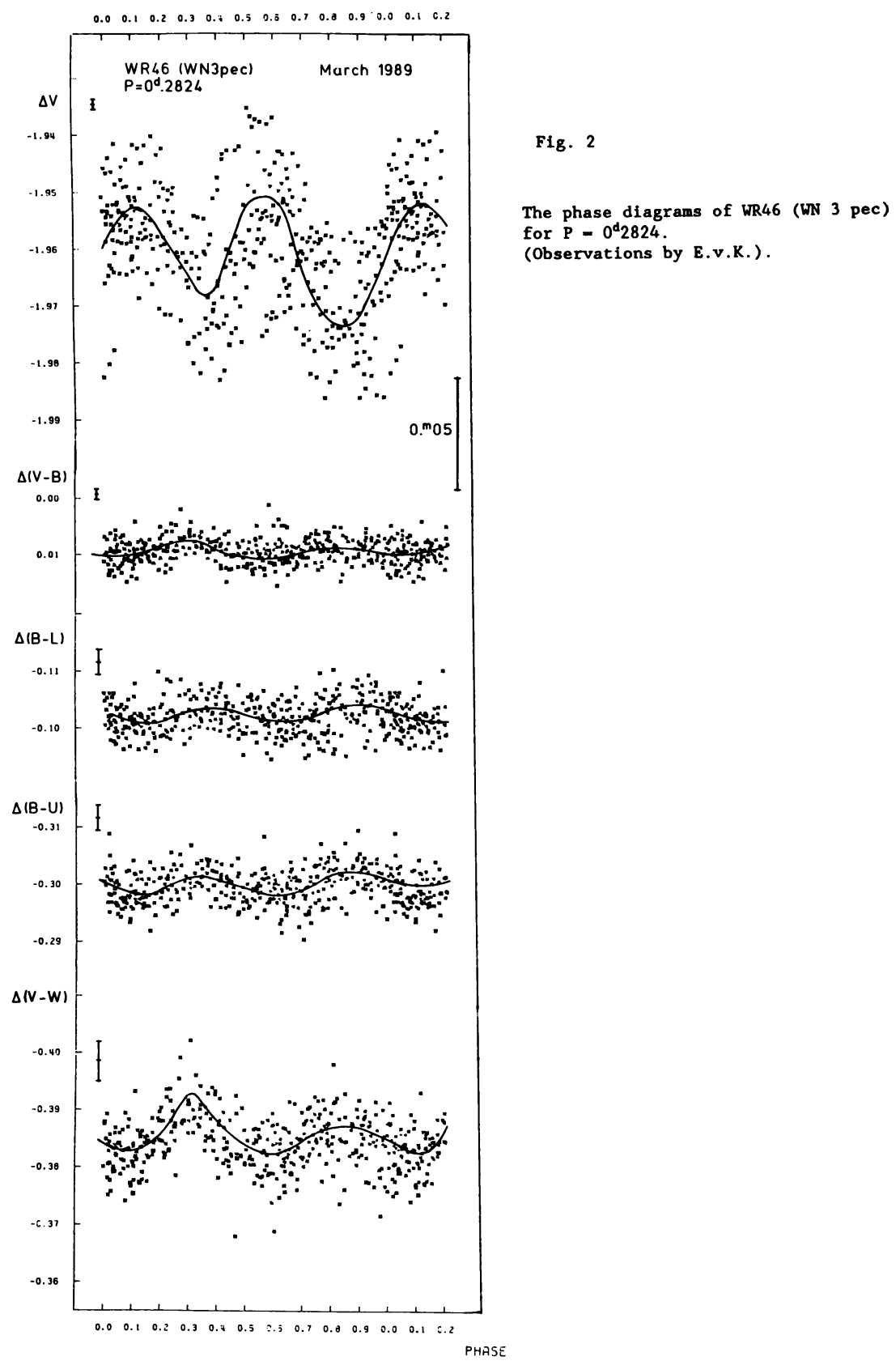



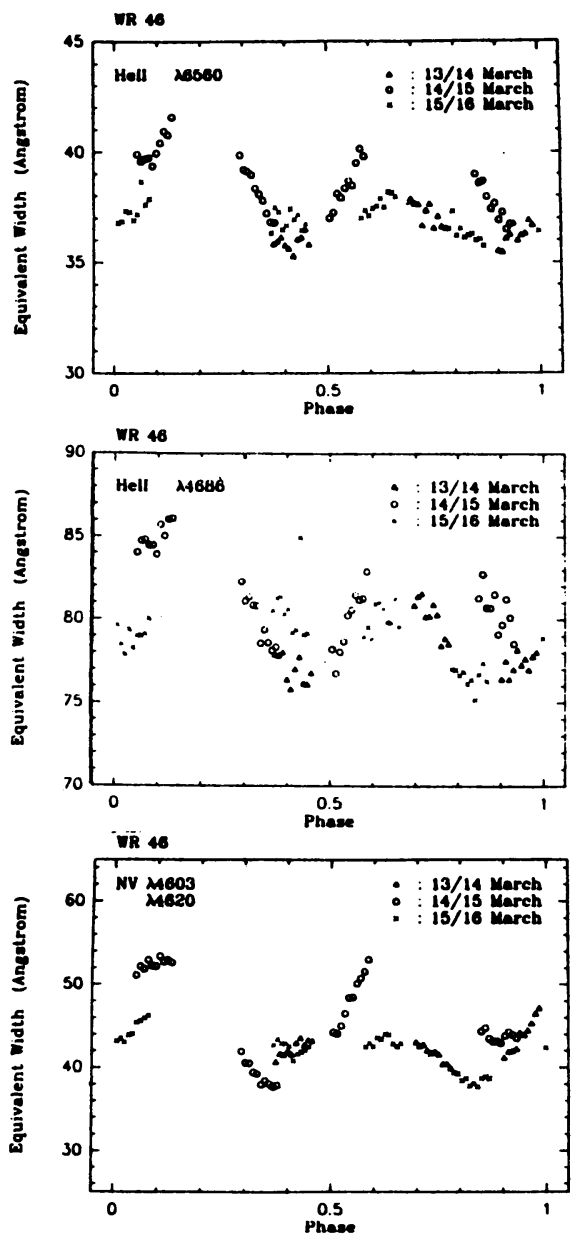

Fig. 3 The variation of the equivalent width for a few proninent lines of WR46 as a function of the phase.

Figure 3 shows for a few prominent lines the variation of the equivalent width in a phase diagram. The total range of the variation per line amount to 10-25\%, (the brightness varies by 108 only). The variations are in phase with the light curve, but they do not vary each night in the same way. Obviously the emission line emitting region shows a stronger distortion than the region which emits the continuum light (the pseudophotosphere). This has the important consequence that the companion presumably revolves within the envelope, while the emission line emitting region closely surrounds both Roche lobes. Besides both regions are apparently subject to geometrical 
changes from cycle to cycle in view of the large scatter in the phase diagrams (Figs. 2 and 3 ). Therefore one may assume that turbulence at a large scale takes place in the pseudo-photosphere as well as in the outer envelope.

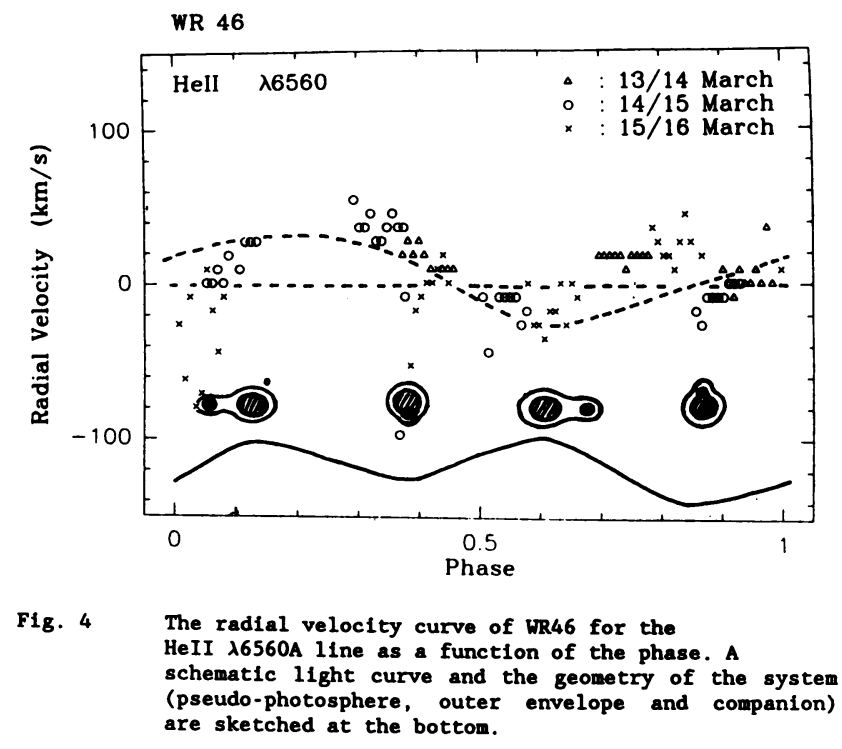

Figure 4 shows the radial velocity curve of the HeII 6560 line in a phase diagram. Also here the scatter is large. The dotted curve should therefore be considered with care. The light curve and the geometry of the system (pseudo-photosphere, outer envelope and companion) are sketched at the bottom.

The dotted curve roughly sketched through the data points, indicates $V_{1}$ sin $\mathrm{i} \sim 40 \mathrm{kms}^{-1}$.

Then with $\mathrm{P}=0^{\mathrm{d}} 2824, a_{1}$ sin $i=1.610^{5} \mathrm{~km}$ and the mass function $\mathrm{f}(\mathrm{M})$ $=0.002$. With $M_{1}+M_{2}=5 M_{\theta}, V_{\text {orbit }}=V_{1}+V_{2}=520 \mathrm{kms}^{-1}$ (see above) and with the aid of the formula $V_{1}=M_{2 /}\left(M_{1}+M_{2}\right) V_{\text {orbit, }}$ we find $M_{1} \leq 4.6 M_{\odot}$ and $M_{2}$ sin $i=0.4 M_{\odot}$.

For $M_{1}+M_{2}=10 M_{\Theta}$ these latter values amount to 9.4 and 0.6 $M_{\theta}$, respectively.

Consequently, if the total mass estimation given above is of the correct order, the mass of the primary lies presumably between 4 and $9 M_{\Theta}$, its radius between 2 and $3 R_{\Theta}$, the mass of the secondary between 0.4 and $0.6 \mathrm{M}_{\theta}$. The latter could then be a white dwarf. However, if we are dealing with a neutron star $\left(\geq 1.4 M_{\Theta}\right)$ then the total mass of the system should lie within the range of $30-40 \mathrm{M}_{\Theta}$. 
According to our binary model the moment with the shallow minimum and the bluest colour corresponds with the companion in front! Based on an analysis of the ratio line flux/continuum flux in $V$ and $B$ band-passes as a function of the phase, a correction of the V-B colour index was possible. The effect of the line flux was subtracted from the photometric flux, leaving a pure continuum variation. It appeared that the amplitude of the colour curve V-B doubled up to $0^{\mathrm{m}} 02$.

\subsection{WR50 (WC6a)}

WR50 $\left(V_{J} \sim 11.9\right)$ was observed relative to the same comparison star and in the same nights as WR46 (sect. 4.1). The schematic light and colour curves for the nine nights in March 1989 are shown together in Fig. 5 .

Due to the low signal to noise ratio in the $\mathrm{W}$ band pass ( $\lambda_{\text {eff }}=$ 3235 A), no U-W curve can be given. In February 1989 the variations showed a smaller range.

One set of typical light and colour curves obtained in 1988 (van Genderen et al., 1990) is shown also for comparison purposes (dotted curve). In 1989 colours are reddest at minimum light. The $\mathrm{B}-\mathrm{L}$ curve (for B $\lambda_{\text {eff }}=4300 \mathrm{~A}$, for $\mathrm{L} \lambda_{\text {eff }}=3840 \mathrm{~A}$ ) does not vary much. Due to the fact that each night showed more or less the same trend: bright in the beginning and faint at the end of the night, the period or quasi-period may be close to $1^{\mathrm{d}}$. By shifting the descending branches on each other we obtain $P \sim 1^{d} 06$.

The behaviour of especially the colour V-B is often opposite to that from 1988 (van Genderen et al., 1990): then the star is sometimes blue during minimum light. It is possible that those variations concern another phase of the light curve, if we are dealing with an eclipsing phenomenon, see further.

The largest drop in brightness and reddening of the colours occurs in the night of $19 / 20$ March: the drop amounts to $0^{\mathrm{m}} 2$ in $\mathrm{V}$, $0^{\mathrm{m}} 5$ in $\mathrm{B}$ and $\mathrm{L}$ and even $0^{\mathrm{m}} 9$ in $\mathrm{U}$ !

The simultaneously obtained spectroscopic observations in three nights of March 1989 by one of us (H.E.S.), are partly analyzed and allow some quantitative discussion. Each night about 40 spectra were obtained with integration times of 3 minutes. See for a comparison of a typical WC spectrum and the position of the $\mathrm{V}$ and B band-passes Fig. 7 in van Genderen et al. (1990).

Figure 6 shows for one night the typical trend of the ratio line flux/continuum flux. It appears that the ratio increases for the $V$ and $B$ band passes by 5-10\%, while the amount of light drops (see Fig. 5). Thus the contribution to the descending branch of the light curve in $\mathrm{V}$ and $\mathrm{B}$ is larger for the continuum light. Applying these ratios and the photometric fluxes in the $V$ and $B$ band-passes, it appears that the line fluxes stop declining midway the descending branch.

It is possible that we are dealing with an eclipsing phenomenon. The peculiar and variable characteristics of the 


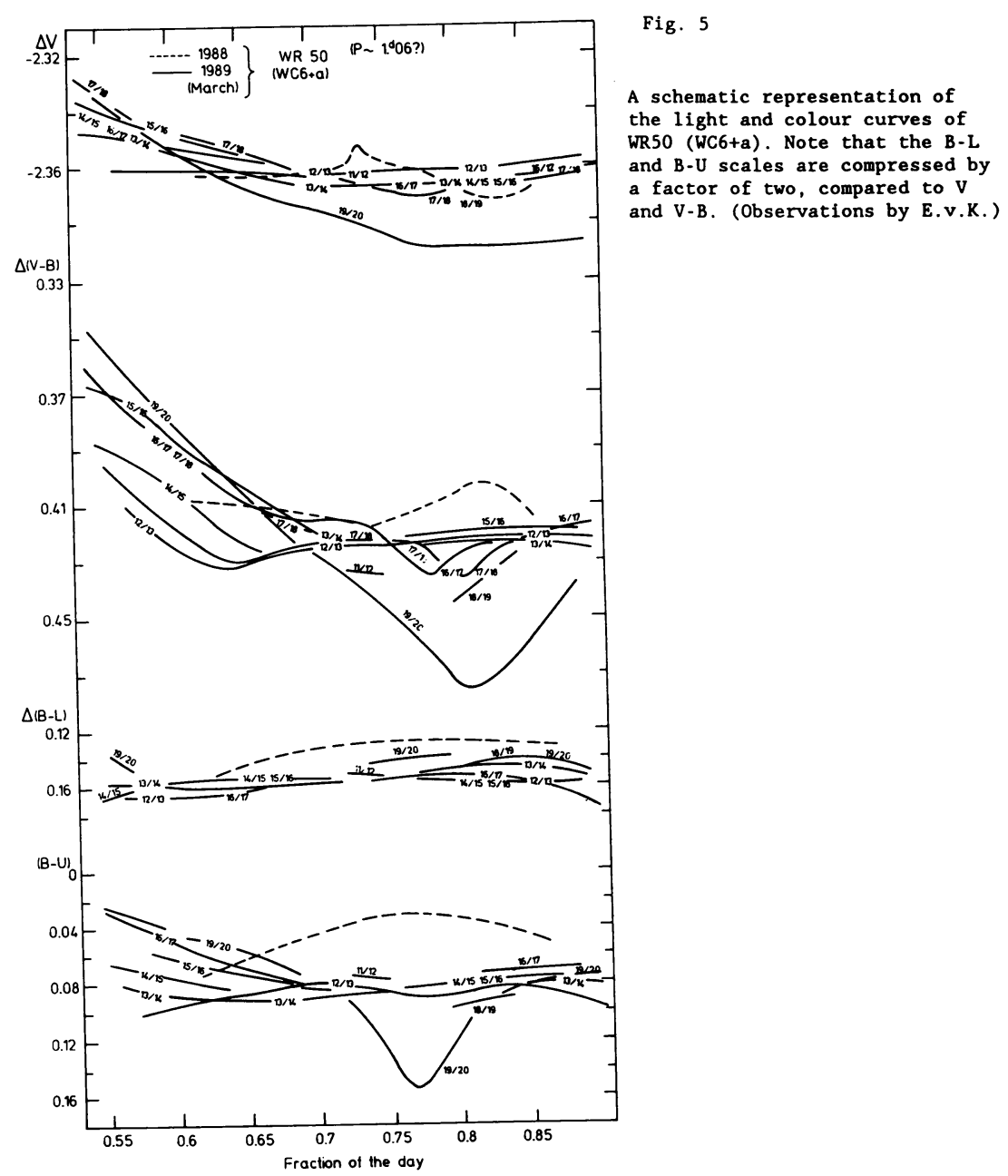

light curve may be the result of an intricate eclipse of (pseudo)-photospheric light and a luminous disk/envelope with variable geometry. Such an eclipse could cause peculiar centerto-limb effects and the peculiar progressive increase of the light amplitudes to shorter wavelengths. The type of the companion is still unknown.

\subsection{WR55 $=$ HD117688 (WN7)}

WR55 $\left(\mathrm{V}_{\mathrm{J}} \sim 10.9\right)$ was observed relative to $\mathrm{HD} 116875\left(\mathrm{~B} 8 \mathrm{~V}, \mathrm{~V}_{\mathrm{J}} \sim 7.8\right.$ ) during nine nights in April and May 1989 by two of us (F.H.A.R. 


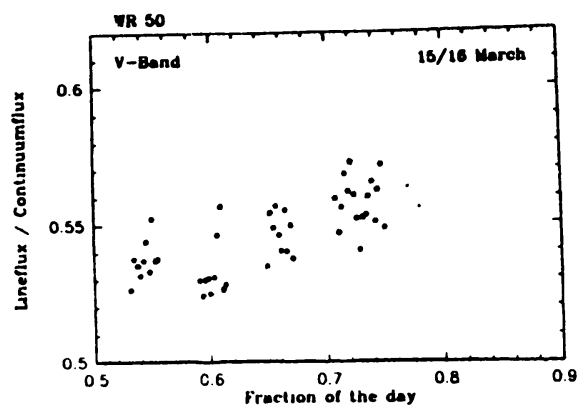

Fig. 6

The trend in one night of the ratio line flux/continuum flux of WR50 for the $V$ and $B$ bandpasses and for the whole spectral range.
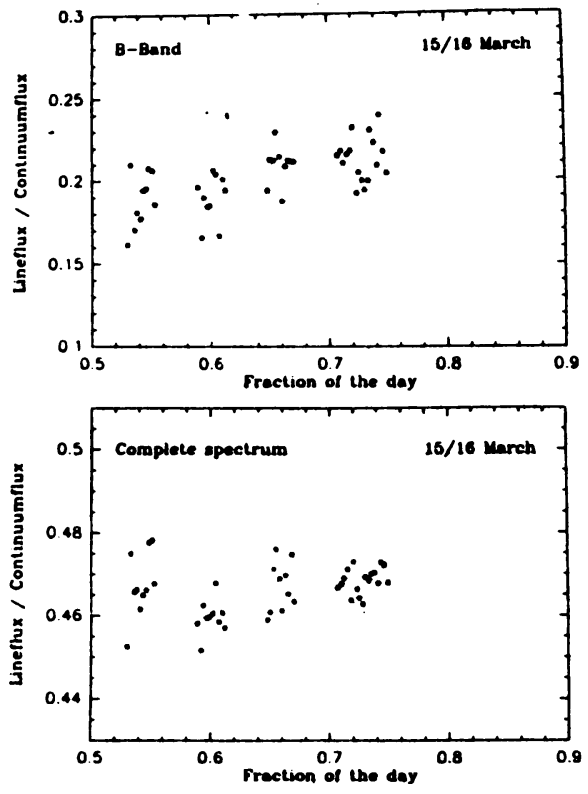

and R.v.d.H., respectively). The period search program was applied between $1^{d}$ and $3^{d}$ (the range in which a possible period should lie) with steps of $0^{d} 02$. There are several candidate periods such like $1^{\mathrm{d}} 22$ and $2^{\mathrm{d}} 56$, but the light curves are not convincing at all. Twice the first mentioned one: $P=2^{\mathrm{d}} 44$ resulted in a peculiar binary type light curve as shown in Fig. 7. The colour $V-B$ is generally slightly redder in the light maxima than in the light minima.

A binary period of this length is not impossible, adopting a radius of $10 R_{\Theta}$ for the WR star and a smaller radius for the component. The adopted total mass lies in the range of 20-50 $\mathrm{M}_{\Theta}$.

Rotation modulation of a deformed WR star and a smaller massive companion is thus possible, but then one has to assume a 


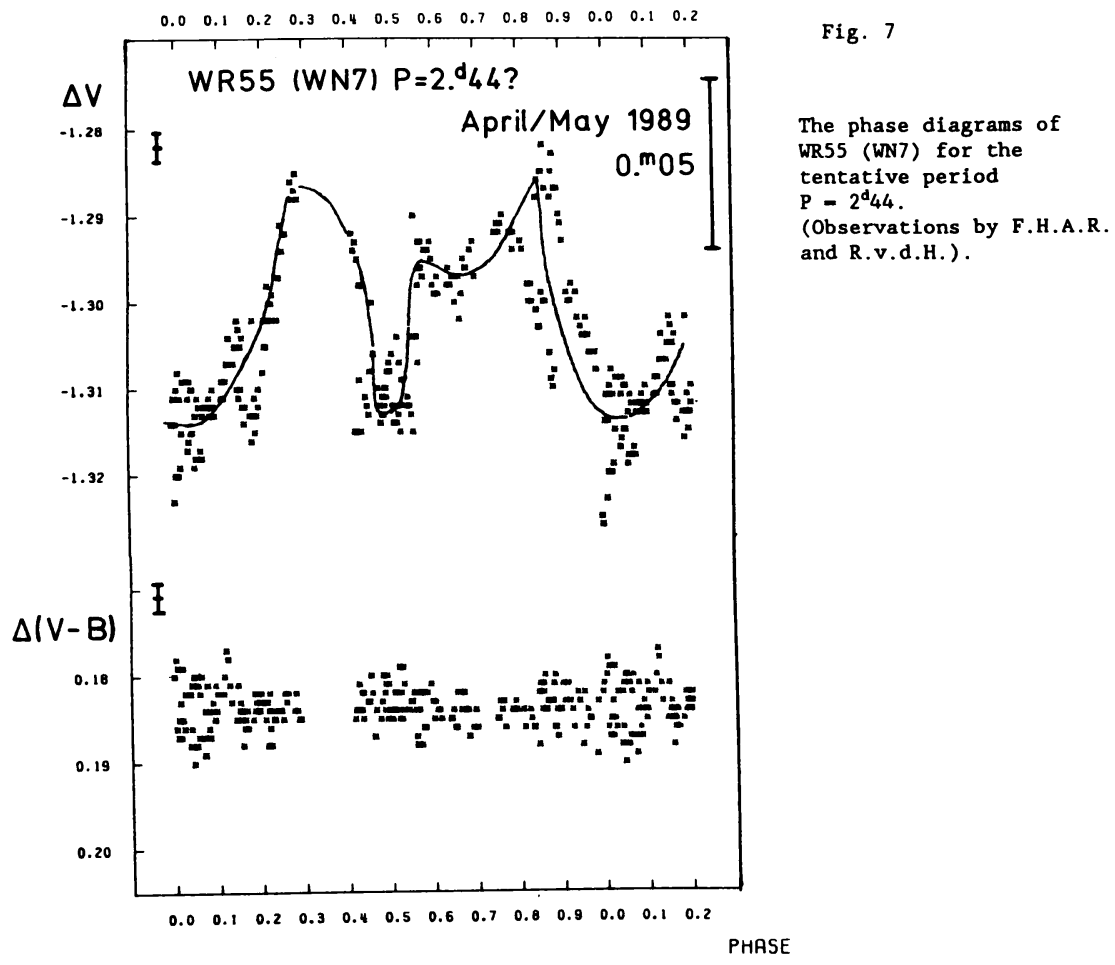

quite peculiar light distribution across the circumference of the WR star.

More observations are planned to increase the number of observations. Until then we have to consider the period as highly uncertain.

4.4 WR86 = HD156327 (WC7), visual companion BOV, sep $\approx 2 "$

WR86 ( $\left.V_{J} \sim 9.3\right)$ was observed relative to HD158528 (A5, $\left.V_{J} \sim 8.4\right)$ during six nights in July 1989 by two of us (L. S. and H.v.W.G.). The spectral type of the companion is determined by Smith (1968), implying that the magnitude is $\sim 1^{\mathrm{m}} 5$ fainter than the WR star (van der Hucht et al., 1988).

The star was also monitored during one night in 1986 showing an oscillation of $\sim 3 \mathrm{~h}$ and an amplitude of $\sim 0^{\mathrm{m} 02}$ in $\mathrm{V}$ (Monderen et a1., 1988). The colour curves are bluer in the light maxima. The same type of variation is exhibited by the new observations. The result is shown in the phase diagrams for $V, B, L, U$ and $W$ of Fig. 8 with $P=0^{d} 1385 \pm 0^{d} 0002$. The scatter is partly intrinsic 


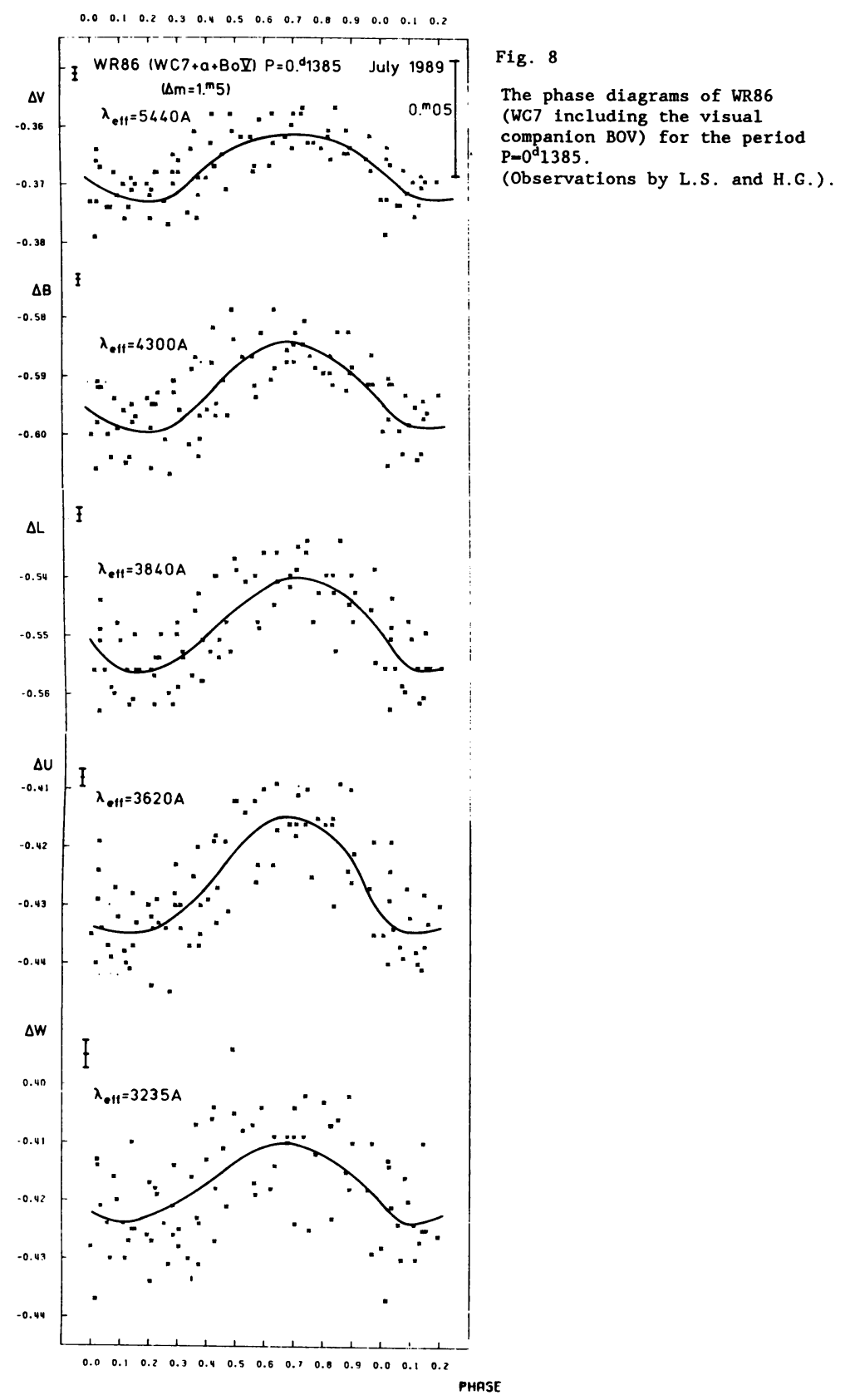


due to the fact that the cycles differ significantly from each other. The maximum amplitude in $\mathrm{V}$ amounts to $\sim 0^{\mathrm{m}} 05$. The colour curves (not shown) are bluer during maximum light except for the $\mathrm{U}-\mathrm{W}$ curve, which is redder. The reason is that the light amplitude in $W$ is smaller than that of the $U$ band pass.

It is possible that the B type companion is the source of this variability, which could then be a $\beta$ Cep star. The length of the period, the shape and variability of the light curves and the size of the amplitude $\left(\sim 0^{\mathrm{m}} 25\right.$ if a correction is applied for the effect of the light of the WR star) agree with that supposition (see the review paper of Lesh and Aizenman (1978)).

However, the fact that the progression of the size of the light amplitude to shorter wavelengths is interrupted by the amplitude in the $\mathrm{W}$ band pass (see for example $\mathrm{BW} \mathrm{Vul}$ in Lesh and Aizenman (1978) and $\tau^{\prime}$ Lup observed by Cuypers (1987), disagrees with the $B$ Cep theory.

New photometry is planned to investigate the variability further. Simultaneous spectroscopy in a few nights is made, but not yet available for a discussion.

\subsection{WR123 $=$ HD177230 (WN8)}

WR123 $\left(V_{J} \sim 11.3\right)$ was observed relative to HD174916 (A0, $\left.V_{J} \sim 7.5\right)$ during 17 nights in September and October 1989 by two of us (R.S.1.P. and R.A.R). The star turned out to be variable with an amplitude of $\sim 0^{\mathrm{m}} 15$.

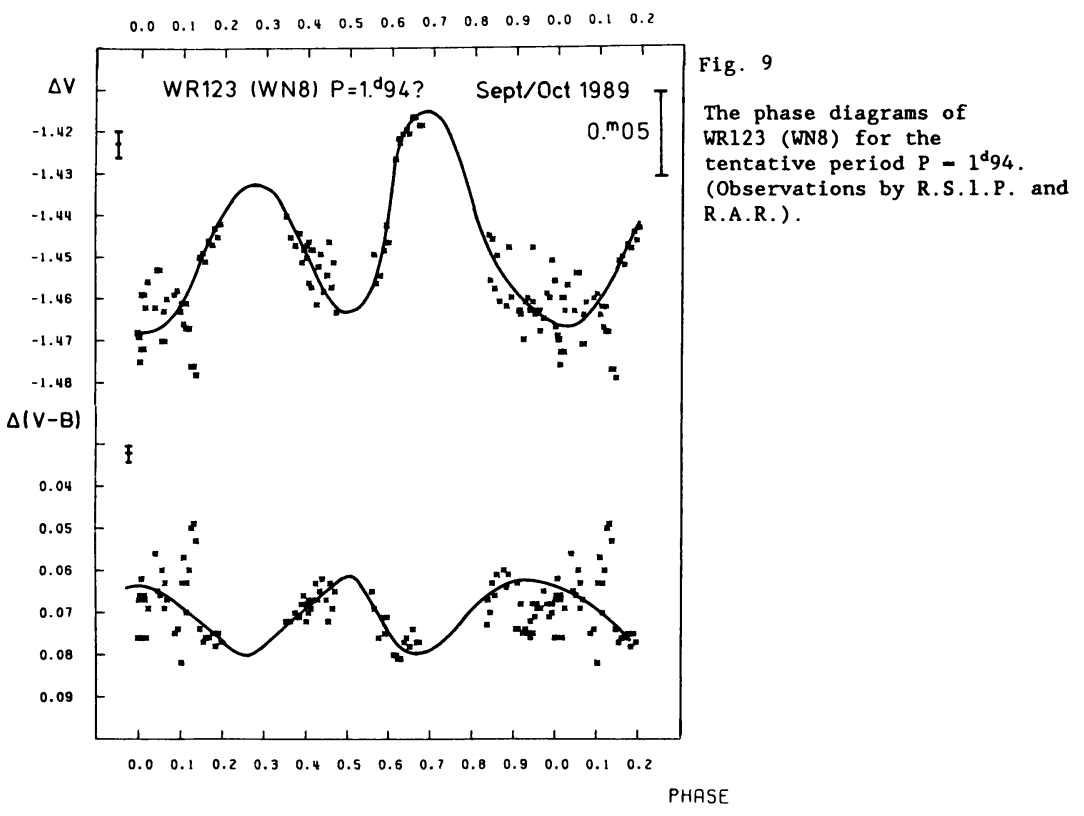


A search of a period was made between $0^{d} 5$ and $2^{d} 5$ with steps of $0^{d} 01$. The best but very tentative result is $P=1^{d} 94$ of which

The peculiar light curve (with two unequal maxima) looks likethe phase diagrams for $\mathrm{V}$ and $\mathrm{V}-\mathrm{B}$ are shown in Fig. 9. that of a binary with an elongated bright companion (the WR stars) similar to WR46 and WR55. Indeed a RV curve revealed by Lamontagne et al. (1983) proofs that a companion is present. They claim that it might be a neutron star. However their tentative period of $1^{\mathrm{d}} 76$ and those of Moffat and Shara (1986): $1^{\mathrm{d}} 76$ or $2^{\mathrm{d}} 37$ do not fit the present material. Whether the period is correct or not, the light minima correspond with the bluer colour in V-B (not so outspoken in the other colour indices), similar to WR46 and WR55, which also have doubly peaked light curves (Figs. 1, 2 and 7).

New observations are planned to investigate the object further, until then the period should be considered with reserve.

\section{Conclusions}

Three of the five variable WR stars discussed here show light curves which presumably reflect a rotation modulation of an elongated star (WR46, WR55 and WR123). The presence of a smaller companion for each of them is suggested. For WR123 (WN7) this seems to be the case indeed in view of its variable radial velocity (Lamontagne et al., 1983).

The period of revolution for WR46 (WN3pec) implicates that it is the shortest period known among the WR binaries. The tentative radial velocity curve of the HeII $6560 \mathrm{~A}$ line indicates a mass function $f(M)=0.002$. The orbital velocity is at least $500 \mathrm{kms}^{-1}$ and the mass and radius for the WR component likely lie in the range of 4-9 $M_{\theta}$ and of 2-3 $R_{\theta}$, respectively. The companion, presumably a white dwarf, could have a mass in the range 0.4-0.6 $M_{\theta}$. However, if it is a neutron star $\left(-1.4 M_{\Theta}\right)$ then the total mass of the system should lie in the range $30-40 \mathrm{M}_{\ominus}$. The variation of the light curve may be caused by secondary effects such like a fast varying geometry of the regions from which we receive the continuum and the line emission. The equivalent widths of the most prominent lines show a phase dependent variation of 10-258, indicating that the line emitting region (the envelope) is more distorted than the continuum emitting region (the pseudophotosphere). Possibly this envelope surrounds both Roche lobes.

Among the two other (WC type) objects, WR50 (WC7) shows a light variation with a time scale of presumably $-1^{\mathrm{d} .06}$ and a light amplitude which increases strongly to the short wavelengths: $\sim 0^{\mathrm{m}} 2$ in $\mathrm{V}, \sim 0^{\mathrm{m}} \mathrm{g}$ in $\mathrm{U}$. A peculiar type of eclipse is possible.

Finally WR86 (WC7) shows a light curve which looks like that of a $B$ Cep star. If this interpretation is correct the observed variation must be that of the visual B type companion.

We can conclude that the present material does not give us a reason to believe that any of the variations are caused by the 
photosphere of the WR star itself. Duplicity and other sources of a "higher order" (Sect. 1) are the main cause.

\section{References}

Cherepashchuk, A.M., Eaton, J.A., Khaliullin, K.H.F.: 1984, Astrophys. J. 281, 774

Cuypers, J.: 1987, Astron. Astrophys. Supp1. 69, 445

van Genderen, A.M., van der Hucht, K.A.: 1986, Astron. Astrophys. 162,109

van Genderen, A.M., van der Hucht, K.A., Steemers, W.J.G.: 1987, Astron. Astrophys. 185,131

van Genderen, A.M., van der Hucht, K.A., Bakker, P.R.: 1989, Astron. Astrophys. 224, 125

van Genderen, A.M., van der Hucht, K.A., Larsen, I.: 1990, Astron. Astrophys. $\underline{229}, 123$

Hillier, D.J.: 1987, Astrophys. J. Suppl. 63,947

Hamann, W.R., Schmutz, W., Wessolowski, U.: 1988, Astron. Astrophys. 194, 190

van der Hucht, K.A., Conti, P.S., Lundström, I., Stenholm, B.: 1981, Space Science Rev. 28, 227

van der Hucht, K.A., Hidayat, B., Admiranto, A.G., Supelli, K.R.: 1988, Astron. Astrophys. 199, 217

van der Hucht, K.A., van Genderen, A.M., Bakker, P.R.: 1990, Astron. Astrophys. $\underline{228}, 108$

Lamontagne, R., Moffat, A.F.J., Seggewiss, W.: 1983, Astrophys. J. $\underline{269}, 96$

Langer, N.: 1989, Astron. Astrophys. 210, 93

Lesh, J.R., Aizenman, M.L.: 1978, Ann. Rev. Astron. Astrophys. $\underline{16}, 215$

Lub, J., Pel, J.W.: 1977, Astron. Astrophys. 54, 137

Maeder, A., Meynet, G.: 1987, Astron. Astrophys. 182, 243

Moffat, A.F.J., Shara, M.M.: 1986, Publ. Astron. Soc. Pacific 92 , 952

Monderen, P., de Loore, C.W.H., van der Hucht, K.A., van Genderen, A.M.: 1988, Astron. Astrophys. 195, 179

Smith, L.F.: 1968, Monthly. Not. Roy. Astron. Soc. 138, 109

Sterken, C.: 1977, Astron. Astrophys. 57, 361

Vreux, J.M.: 1987, Proc. Workshop C. de Jager: Instabilities in Luminous Early Type Stars, eds. H.J.G.L.M. Lamers and C.W.H. de Loore, Reide1, Dordrecht, p. 81 


\section{DISCUSSION}

Niemela: How accurately is the distance of WR46 known? With such a short period, could it be one of those "naked planetaries"?

van Genderen: The distance is not known very well. It is not a member of a cluster or associations. Van der Hucht et al. (1988) estimate a distance of $3.4 k p c$, for an adopted average absolute visual magnitude $\mathrm{M}_{v}(\mathrm{WN} 3)=-2.8$.

Schulte-Ladbeck: You mentioned that the line emitting region in WR46 is more distorted than the continuum region. Could you please specify or describe this in more detail?

van Genderen: The amount of light is proportional with the visible surface. The light curve shows a distortion of $10 \%$ (amplitude is 0.1 ), that is to say, if we face the points of the elongated star (surface $\sim \pi a^{2}$ ), we receive $10 \%$ less light than if the star is seen from aside (surface $\sim \pi a b$ ). Consequently the ratio between the two is $\sim b / a$. Since the amplitude of the curves representing the variation of $W_{\lambda}$ ranges from $10-25 \%$, the distortion $b / a$ also ranges up to $25 \%$. Therefore the envelope from which we receive the emission lines must be more distorted than the pseudophotosphere from which we receive the continuum light. Therefore we suggest that the envelope also surrounds the companion.

Cassinelli (question directed to Maeder): Your conclusion that pulsation is not occurring in WR stars is a very important one in regard to possible mechanisms for driving mass loss. I recall from Maeder et al. (1983) that interior theorists argue that WR stars lose sufficient mass by a wind to remain quasi-homogeneous. I can think of no way for the deep interior to affect the mass loss except by way of pulsation. Your conclusion therefore indicates that one cannot use the quasi-homogeneity argument to derive mass loss rates.

van Genderen: My first remark "Do variations of WR photospheres exist?" gives the wrong impression that I do not believe in them. If they exist, it proves that the envelopes are so optically thick, that they cannot be detected at all.

Maeder: If you do not maintain quasi-homogeneity by high mass loss you will have the WR star much too luminous, as shown by Hamann. The model would be too luminous. So in order to have WR models at the location found by Hamann et al., you need to lose a lot of mass. And this is a way to assure homogeneity and of course if there are additional mixing mechanisms this would also favour the homogeneity of the star. 


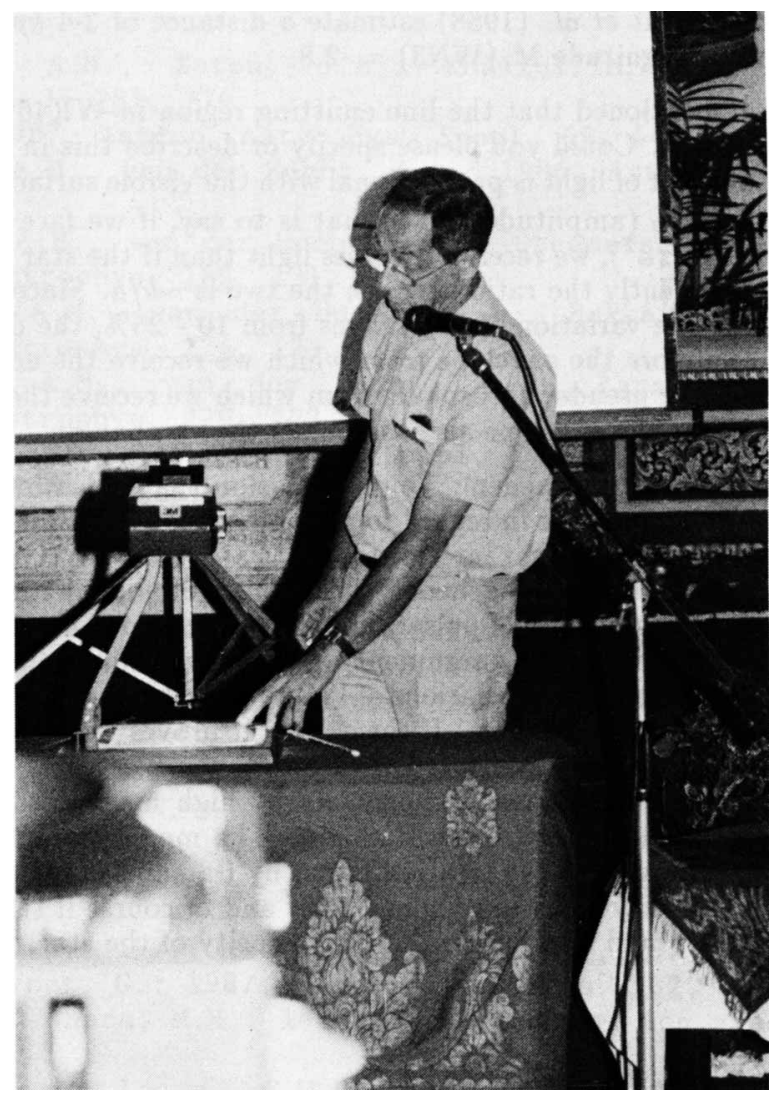

van Genderen 